\title{
Effects of FGFR1 Gene Polymorphisms on the Risk of Breast Cancer and FGFR1 Protein Expression
}

\author{
Junqiang Wu ${ }^{\mathrm{a}}$ Yuhang Wang ${ }^{\mathrm{a}}$ Jing Liu ${ }^{\mathrm{b}}$ Qianlin Chen ${ }^{\mathrm{a}} \quad$ Da Pang \\ Yongdong Jianga \\ aDepartment of Breast Surgery, The Third Affiliated Hospital of Harbin Medical University, Harbin, \\ bDepartment of Anesthesiology, The Second Affiliated Hospital of Harbin Medical University, Harbin, \\ China
}

\section{Key Words}

FGFR1 • Gene polymorphisms • Breast cancer • Susceptibility • Protein expression

\begin{abstract}
Background/Aims: Fibroblast growth factor receptor 1 (FGFR1) is widely considered to play an important role in mammary carcinogenesis. Some common variants in FGFR1 might be associated with its expression, and further affect breast cancer risk. The aim of this study was to investigate effects of single-nucleotide polymorphisms (SNPs) in FGFR1 on breast cancer susceptibility and FGFR1 protein expression. Methods: SNPs rs17182023, rs17175624 and rs10958704 in FGFR1 were genotyped in 747 breast cancer cases and 716 healthy controls by SNaPshot method. The associations between SNPs and breast cancer were examined by logistic regression. Immunohistochemistry(IHC) was used to detect FGFR1 protein expression, and the association of FGFR1 polymorphisms with its protein expression was analyzed by Pearson's chi-square test. Additionally, Cox regression and Kaplan-Meier analysis was used to evaluate the association between FGFR1 protein expression and breast cancer prognosis. Results: The minor allele of rs17182023 in FGFR1 was significantly associated with reduced breast cancer risk, with an odds ratio of $0.800(95 \% \mathrm{CI}=0.684-0.935)$. No significant associations were detected between other SNPs and breast cancer. Moreover, rs17182023 was correlated to FGFR1 protein expression $(P=0.006)$, and patients with high FGFR1 protein expression tended to have poor outcomes. Conclusions: SNP rs17182023 was correlated to reduced breast cancer risk, and was associated with FGFR1 protein expression. High FGFR1 protein expression was an independent risk factor of breast cancer, and resulted in poor prognosis.
\end{abstract}




\section{Introduction}

Breast cancer is considered to be one of the most common malignancies in women [1]. It is widely recognized that breast cancer is a complex, systemic disease which associated with multiple genetic and environmental factors. According to a large number of studies, activation of tyrosine kinase growth factor receptors is proved to be a pervasive oncogenic event in breast cancer. The fibroblast growth factor receptors (FGFRs) belong to receptor tyrosine kinase (RTK) superfamily, and the amino-acid sequences of FGFRs are highly conserved. They function in a wide range of biological process, such as development controlling, angiogenesis, wound repair and endocrine functions [2-4]. Moreover, preclinical evidences have demonstrated that activation of FGFR signalling pathways, which may be caused by point mutations, amplifications and translocations, play significant roles in tumor development and progression [5, 6].

Multiple genetic alterations in FGFRs have been found in breast cancer. An intronic variant in FGFR2 was one of the first SNPs being identified [7, 8], and was recognized as a breast cancer susceptibility locus, especially in estrogen receptor (ER)-positive disease. Other variants in FGFRs and the downstream genes in the FGF pathway may also be associated with breast carcinogenesis. For instance, amplification of FGFR1 is identified in up to $10 \%$ of all breast cancer and $16 \%$ to $27 \%$ of luminal type B breast cancer cases, and it is correlated with endocrine therapy resistance and poor prognosis $[9,10]$. Studies from breast cancer cell lines and mouse models also support a role for FGFR1 amplification in mammary oncogenesis $[11,12]$. The aberrant activation of FGFR1 in mouse or human mammary cell lines resulted in increased cell proliferation, survival and invasion, confirming the carcinogenic nature of FGFR1 signalling.

Currently, as the carcinogenic effect of FGFR1 is widely accepted, an increasing number of studies have been working on it, since FGFR1 could be a potential diagnostic and (or) prognostic biomarker. To date, several studies about FGFR1 genetic polymorphisms have been carried out, trying to find SNP loci which could be a predictor for breast cancer risk. However, according to the previous studies, few loci were confirmed to be significantly correlated with breast cancer susceptibility [13]. Thus, we carried out this case-control study. Three SNPs in FGFR1 gene: rs17182023, rs17175624 and rs10958704, were picked out from the SNP databases, and all of them were intronic variants near the 5' end of FGFR1. According to the analyses, These variants were potentially functional SNPs, which might alter the expression of FGFR1. Therefore, we genotyped the three SNPs in breast cancer cases and healthy controls, trying to identify their effect on FGFR1 protein expression, revealing the associations between these common genetic variants and breast cancer risk, and moreover, confirming the impacts of FGFR1 protein expression on disease characters and prognosis of breast cancer.

\section{Materials and Methods}

\section{Subjects}

There were 747 breast cancer cases and 716 healthy controls included in this study. The cases were patients with sporadic breast cancer from the Department of Breast Surgery at the Third Affiliated Hospital of Harbin Medical University from September 2008 to December 2011. The patients were diagnosed based on their surgical and pathological evaluation, and the information of their disease was obtained from the medical files (Table 1).
Table 1. The demographic characteristics of 747 breast cancer cases and 716 controls. Note: Data presented as the mean \pm standard deviation or number ( $\%$ of total number)

\begin{tabular}{lccc}
\hline Characteristics & Case $(\mathrm{n}=747)$ & Controls $(\mathrm{n}=716)$ & $\mathrm{P}$ \\
\hline Age & $49.91 \pm 10.28$ & $49.55 \pm 10.61$ & 0.508 \\
Body mass index, BMI & $24.03 \pm 3.44$ & $23.32 \pm 3.13$ & $<0.001$ \\
Age at menarche & $15.37 \pm 1.80$ & $15.61 \pm 1.94$ & 0.015 \\
Age at first birth & $23.79 \pm 6.11$ & $24.00 \pm 7.41$ & 0.556 \\
Breastfeeding duration & $16.59 \pm 13.77$ & $11.43 \pm 7.48$ & $<0.001$ \\
Menopausal status & & & \\
Pre-menopausal & $415(55.6)$ & $457(63.8)$ & 0.001 \\
Post-menopausal & $332(44.4)$ & $259(36.2)$ & \\
Family history & & & \\
No & $622(83.3)$ & $607(84.8)$ & 0.431 \\
Yes & $125(16.7)$ & $109(15.2)$ & \\
\hline
\end{tabular}


Wu et al.: FGFR1 Polymorphisms Affect Breast Cancer Risk

The control group consisted of women of Han origin living in Harbin, in northeastern China. The individuals who were selected had no history of cancer, and their age and ethnicity is matched with the cancer patients. Furthermore, the participants were interviewed respectively to collect information on demographic characteristics, and each of them provided $5 \mathrm{ml}$ of venous blood. The study was approved by the ethics committee of Harbin Medical University.

\section{SNP selection and genotyping}

In this study, a combined analysis of functional significance and Tag SNP strategies was utilized to select three potentially functional SNPs in the FGFR1 gene from the dbSNP and HapMap databases. The minor allele frequencies (MAF) of these SNPs were greater than 5\%, and the pair-wise $r^{2}$ values were greater than 0.8. Genomic DNA was isolated from EDTA anti-coagulated whole blood using the AxyPrep Blood Genomic DNA Miniprep Kit (Axygen Biotechnology, Tewksbury, MA, USA). We performed the SNaPshot SNP assay to checkout the polymorphisms at the three SNPs. The resulting data were analyzed with GeneMapperTM 4.0 software (Applied Biosystems, Foster City, CA, USA).

\section{IHC}

IHC was used to detect FGFR1 protein expression of 348 breast cancer tissues which were randomly selected from the 747 breast cancer cases. IHC staining was performed using a Benchmark XT auto-stainer (Ventana Medical Systems Inc., Tucson, AZ, USA), and the FGFR1 antibody (ab10646, Abcam, Cambridge, UK) was diluted to 1:2000. IHC was proceeded as follows. Briefly, 4-micron thick tissue sections were cut in microtome, deparaffinized in xylene, rehydrated through graded ethanol (100\% and 95\%), and rinsed in water. Subsequently, the sections were subjected to heat-induced antigen retrieval, and finally loaded onto the Benchmark auto-stainer and the detection was performed using the I-View detection kit (Ventana Medical Systems Inc., Tucson, AZ, USA). IHC of ER, PR, HER2, Ki67 and P53 (ZSBG-BIO: ZM-0104, ZM-0215, ZM-0065, ZM-0165, ZM-0405) was performed similarly to the description above. Protein expression of FGFR1 was quantified as a percentage (range 0-100\%) of positive cells present among all tumour cells presented. A cut-off value of $10 \%$ was selected for FGFR1 protein expression. Above $10 \%$ was defined as "high expression" and below as "low expression" [14] (Fig. 1). IHC staining for ER and PR was considered positive if $\geqslant 1 \%$ of tumour cell nuclei were stained [15]. Expression of HER2 was evaluated with the HercepTest kit (Dako) and scored as $0,1+, 2+$ and $3+$. Scores of 0 and $1+$ were determined as negative, and $3+$ was determined as positive [16]. The intrinsic subtypes were determined using the molecular typing method [17]. Each of the sections was scored by two observers, who were blinded to patients' medical information, and disagreement between the observers was solved by discussion.

\section{Statistical analysis}

The genotype frequencies were tested for Hardy-Weinberg equilibrium using the chi-square test. Differences between the cases and controls in demographic characteristics were evaluated by Pearson chisquare test (for categorical variables) or Student's t-test (for continuous variables). Associations between genotypes and breast cancer risk were estimated by computing odds ratios (ORs) and 95\% confidence intervals (CIs) from logistic regression. To eliminate the effect of confounding factors, we corrected for multivariate analysis and adjusted for age, body mass index (BMI), breastfeeding duration, age at menarche and menopausal status. Associations between disease characteristics and patient genotypes were evaluated by Pearson chi-square test. Differences in protein expression and genotypes and associations between FGFR1 protein expression and clinicopathological variables were analyzed with a Pearson chi-square test. Spearman rank correlation analysis was employed to test the linear correlations. Cox proportional hazards model was performed to evaluate prognostic

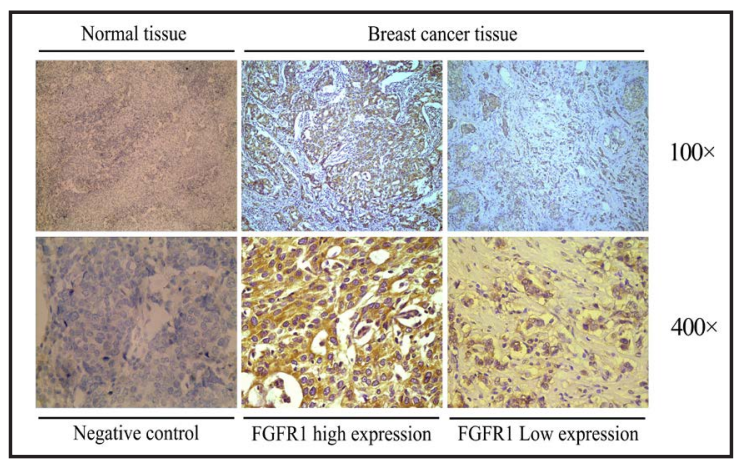

Fig. 1. Representative sections of FGFR1 protein expression in breast cancer tissue and adjacent normal tissue. 
Wu et al.: FGFR1 Polymorphisms Affect Breast Cancer Risk

factors of breast cancer by measuring risk ratios and CIs. Kaplan-Meier analysis was used to analyze the overall survival (OS). The value of $P<0.05$ was considered to be of statistical significance. All the statistical analysis was computed by SPSS 13.0 (SPSS Inc., Chicago, IL).

\section{Results}

\section{Subject characteristics}

The 747 breast cancer cases and 716 healthy controls were similar in age at interview, age at first birth and family history (Table 1), but otherwise show significant differences. In contrast to the controls, the cases tended to have higher body mass index (BMI), earlier age at menarche, longer breastfeeding duration, and higher proportion of postmenopausal individuals $(\mathrm{P}$ $<0.05$ ).

\section{Correlations between} SNPs and breast cancer susceptibility

This study involved three SNPs in FGFR1 gene: rs17182023, rs17175624 and rs10958704, and the distributions of alleles and genotypes were exhibited in Table 2. The genotype frequencies of the SNPs conform to Hardy-Weinberg equilibrium. According to the table, the cases tend to carry less minor allele of rs17182023 compared with controls. But for the other two loci, there were no significant differences in allele and genotype distributions between the cases and controls.

We performed a logistic linear regression to investigate the correlation between the SNPs and breast cancer susceptibility. Results showed that the minor allele of rs17182023 in the FGFR1 gene had a great tendency to reduce the risk of breast cancer, with an OR of $0.785(95 \% \mathrm{CI}$ $=0.676-0.911, P=0.001$ ). We further analyzed the effect of genotypes under two genetic models (Table

Table 2. Genotype distributions, odds ratios (OR) and 95\% confidence intervals (CI) for the association between breast cancer susceptibility loci of FGFR1 in 747 breast cancer cases and 716 controls. Note: ${ }^{a}$ Adjusted for age, BMI, breastfeeding duration, age at menarche and menopausal status

\begin{tabular}{|c|c|c|c|c|c|c|}
\hline Genotype & Case $(\mathrm{n}=747)$ & Control (n=716) & OR (95\% Cl) & & OR $(95 \% \text { CI })^{\mathrm{r}}$ & \\
\hline \multicolumn{7}{|l|}{ rs17182023 } \\
\hline GG & $317(42.4)$ & $248(34.6)$ & 1.000 & & 1.000 & \\
\hline GA & $333(44.6)$ & $349(48.7)$ & $0.746(0.597-0.934)$ & 0.010 & $0.755(0.598-0.953)$ & 0.018 \\
\hline AA & $97(13.0)$ & $119(11.6)$ & $0.638(0.465-0.874)$ & 0.005 & $0.665(0.478-0.924)$ & 0.015 \\
\hline A allele & $527(35.3)$ & $587(41.0)$ & $0.785(0.676-0.911)$ & 0.001 & $0.800(0.684-0.935)$ & 0.005 \\
\hline GA+AA & $430(57.6)$ & $468(65.4)$ & $0.719(0.582-0.888)$ & 0.002 & $0.733(0.588-0.913)$ & 0.006 \\
\hline \multicolumn{7}{|l|}{ rs17175624 } \\
\hline $\mathrm{CC}$ & $346(46.3)$ & $344(48.0)$ & 1.000 & & 1.000 & \\
\hline $\mathrm{CA}$ & $313(41.9)$ & $301(42.0)$ & $1.034(0.832-1.285)$ & 0.764 & $1.072(0.854-1.344)$ & 0.551 \\
\hline & & & $1.232(0.871-1.742)$ & 0.237 & $1.224(0.853-1.758)$ & \\
\hline Aallele & $489(32.7)$ & $443(30.9)$ & $1.086(0.930-1.269)$ & 0.298 & $1.097(0.933-1.291)$ & 0.262 \\
\hline & $401(53.7)$ & $372(52.0)$ & $1.072(0.873-1.316)$ & 0.509 & $1.101(0.889-1.364)$ & 0.378 \\
\hline rs10958704 & & & & & & \\
\hline GA & $318(42.6)$ & $300(41.9)$ & $1.063(0.855-1.321)$ & 0.581 & $1.097(0.874-1.376)$ & 0.426 \\
\hline $\mathrm{AA}$ & $87(11.6)$ & $73(10.2)$ & $1.195(0.846-1.688)$ & 0.311 & $1.192(0.831-1.710)$ & 0.339 \\
\hline Aallele & $492(32.9)$ & $446(31.1)$ & $1.086(0.929-1.268)$ & 0.301 & $1.096(0.932-1.289)$ & 0.267 \\
\hline GA+AA & $405(54.2)$ & $373(52.1)$ & $1.089(0.887-1.337)$ & 0.416 & $1.115(0.900-1.382)$ & 0.318 \\
\hline
\end{tabular}

Table 3. The association between SNP locus rs17182023 and clinicopathological characteristics of breast cancer. Note: ${ }^{a}$ Under dominate model, bunder co-dominate model. LNM: lymph node metastasis, ER: estrogen receptor, PR:

\begin{tabular}{|c|c|c|c|c|c|c|}
\hline Clinico-pathological characteristics & Cases & GG(\%) & $\begin{array}{c}\text { rs17182023 } \\
\text { GA(\%) }\end{array}$ & $\mathrm{AA}(\%)$ & $\mathrm{Pa}$ & $\mathrm{Pb}$ \\
\hline \multicolumn{7}{|l|}{ TNM stage } \\
\hline I, II & 532 & $225(42.3)$ & 238(44.7) & $69(13.0)$ & 0.705 & 0.906 \\
\hline III & 143 & $63(44.1)$ & $63(44.1)$ & $17(11.9)$ & & \\
\hline \multicolumn{7}{|l|}{ Tumor size $(\mathrm{cm})$} \\
\hline$\leqslant 2$ & 583 & $253(43.4)$ & $255(43.7)$ & $75(12.9)$ & 0.297 & 0.428 \\
\hline$>2$ & 88 & $33(37.5)$ & $45(51.1)$ & $10(11.4)$ & & \\
\hline \multicolumn{7}{|l|}{ Pathological stage } \\
\hline I, II & 446 & $188(42.2)$ & $200(44.8)$ & $58(13.0)$ & 0.924 & 0.993 \\
\hline III & 120 & $50(41.7)$ & $54(45.0)$ & $16(13.3)$ & & \\
\hline \multicolumn{7}{|l|}{ LNM } \\
\hline Negative & 425 & $178(41.9)$ & $195(45.9)$ & $52(12.2)$ & 0.795 & 0.701 \\
\hline Positive & 294 & $126(42.9)$ & $127(43.2)$ & $41(13.9)$ & & \\
\hline \multicolumn{7}{|l|}{ ER status } \\
\hline Negative & 244 & $103(42.2)$ & $111(45.5)$ & $30(12.3)$ & 0.896 & 0.838 \\
\hline Positive & 447 & $191(42.7)$ & $195(43.6)$ & $61(13.6)$ & & \\
\hline \multicolumn{7}{|l|}{ PR status } \\
\hline Negative & 319 & $144(45.1)$ & 138(43.3) & $37(11.6)$ & 0.202 & 0.335 \\
\hline Positive & 372 & $150(40.3)$ & $168(45.2)$ & $54(14.5)$ & & \\
\hline \multicolumn{7}{|l|}{ HER2 status } \\
\hline Negative & 492 & $206(41.9)$ & $224(45.5)$ & $62(12.6)$ & 0.717 & 0.478 \\
\hline Positive & 119 & $52(43.7)$ & $48(40.3)$ & $19(16.0)$ & & \\
\hline \multicolumn{7}{|l|}{ Ki67 status } \\
\hline Negative & 258 & $116(45.0)$ & $109(42.2)$ & $33(12.8)$ & 0.296 & 0.571 \\
\hline Positive & 428 & $175(40.9)$ & $196(45.8)$ & $57(13.3)$ & & \\
\hline \multicolumn{7}{|l|}{ P53 status } \\
\hline Negative & 499 & $215(43.1)$ & 214(42.9) & $70(14.0)$ & 0.550 & 0.320 \\
\hline Positive & 185 & $75(40.5)$ & $90(48.6)$ & $20(10.8)$ & & \\
\hline \multicolumn{7}{|l|}{ Intrinsic subtypes } \\
\hline Luminal A & 149 & $64(43.0)$ & $67(45.0)$ & 18(12.1) & 0.489 & 0.758 \\
\hline Luminal B & 273 & $110(40.3)$ & $122(44.7)$ & $41(15.0)$ & & \\
\hline HER2-positive & 162 & $69(42.6)$ & $74(45.7)$ & $19(11.7)$ & & \\
\hline Triple-negative & 27 & $15(55.6)$ & $9(33.3)$ & $3(11.1)$ & & \\
\hline
\end{tabular}


2). For rs17182023, the GA and AA genotypes significantly lower the risk of breast cancer compared to GG genotype under codominate model with ORs of $0.746(95 \% \mathrm{CI}=0.597$ $0.934, P=0.010$ ) and 0.638 $(95 \% \mathrm{CI}=0.465-0.874, P$ $=0.005$ ) respectively, and the result under dominate model was also of statistical significance $(O R=0.719$, $95 \% \mathrm{CI}=0.582-0.888, P$ $=0.002)$. However, no significant associations were found between the other two SNPs and breast cancer susceptibility.

After adjusting for age, BMI, breastfeeding duration, age at menarche and menopausal status, the results were similar to the above. The minor allele of rs17182023 was associated with reduced breast cancer risk $(\mathrm{OR}=$ $0.80095 \% \mathrm{CI}=0.684-0.935$, $P=0.005$ ), and there were also significant correlations between the GA and AA genotypes and lower risk of breast cancer compared with the GG genotype under co-dominate model $(\mathrm{OR}=$ $0.755,95 \% \mathrm{CI}=0.598-0.953$, $P=0.018 ; \mathrm{OR}=0.665,95 \% \mathrm{CI}$ $=0.478-0.924, P=0.015$, respectively) and dominate model $(\mathrm{OR}=0.733,95 \% \mathrm{CI}$ $=0.588-0.913, P=0.006$ ).

\section{Correlations} between SNPS and clinicopathological characteristics

Next, we analyzed the effect of SNP rs17182023, rs17175624 and rs10958704 on a series of clinico-pathological characteristics in the 747 breast cancer cases, including lymph node metastasis, tumor size, tumorgrade, clinic stage, and the status of estrogen receptor (ER) or progesterone
Table 4. The association between SNP locus rs17175624 and clinicopathological characteristics of breast cancer. Note: ${ }^{a}$ Under dominate model, ${ }^{b}$ under co-dominate model

\begin{tabular}{|c|c|c|c|c|c|c|}
\hline Clinico-pathological characteristics & Cases & $\mathrm{CC}(\%)$ & $\begin{array}{c}\text { rs17175624 } \\
\text { CA(\%) }\end{array}$ & $\mathrm{AA}(\%)$ & $\mathrm{Pa}$ & $\mathrm{Pb}$ \\
\hline \multicolumn{7}{|l|}{ TNM stage } \\
\hline I, II & 532 & $244(45.9)$ & $225(42.3)$ & $63(11.8)$ & 0.162 & 0.375 \\
\hline III & 143 & $75(52.4)$ & $53(37.1)$ & $15(10.5)$ & & \\
\hline \multicolumn{7}{|l|}{ Tumor size $(\mathrm{cm})$} \\
\hline$\leqslant 2$ & 583 & $272(46.7)$ & $245(42.0)$ & $66(11.3)$ & 0.433 & 0.603 \\
\hline$>2$ & 88 & $45(51.1)$ & $32(36.4)$ & $11(12.5)$ & & \\
\hline \multicolumn{7}{|l|}{ Pathological stage } \\
\hline I, II & 446 & $216(48.4)$ & $177(39.7)$ & $53(11.9)$ & 0.731 & 0.564 \\
\hline III & 120 & $56(46.7)$ & $53(44.2)$ & $11(9.2)$ & & \\
\hline \multicolumn{7}{|l|}{ LNM } \\
\hline Negative & 425 & $189(44.5)$ & $189(44.5)$ & $47(11.1)$ & 0.311 & 0.301 \\
\hline Positive & 294 & $142(48.3)$ & $114(38.8)$ & $38(12.9)$ & & \\
\hline \multicolumn{7}{|l|}{ ER status } \\
\hline Negative & 244 & $112(45.9)$ & $103(42.2)$ & $29(11.9)$ & 0.963 & 0.981 \\
\hline Positive & 447 & $206(46.1)$ & $186(41.6)$ & $55(12.3)$ & & \\
\hline \multicolumn{7}{|l|}{ PR status } \\
\hline Negative & 319 & $145(45.5)$ & $133(41.7)$ & $41(12.9)$ & 0.782 & 0.869 \\
\hline Positive & 372 & $173(46.5)$ & $156(41.9)$ & $43(11.6)$ & & \\
\hline \multicolumn{7}{|l|}{ HER2 status } \\
\hline Negative & 492 & $226(45.9)$ & $207(42.1)$ & $59(12.0)$ & 0.441 & 0.581 \\
\hline Positive & 119 & $50(42.0)$ & $51(42.9)$ & $18(15.1)$ & & \\
\hline \multicolumn{7}{|l|}{ Ki67 status } \\
\hline Negative & 258 & $105(40.7)$ & $115(44.6)$ & $38(14.7)$ & 0.029 & 0.058 \\
\hline Positive & 428 & $211(49.3)$ & $172(40.2)$ & $45(10.5)$ & & \\
\hline \multicolumn{7}{|l|}{ P53 status } \\
\hline Negative & 499 & $229(45.9)$ & $210(42.1)$ & $60(12.0)$ & 0.890 & 0.970 \\
\hline Positive & 185 & $86(46.5)$ & $76(41.1)$ & $23(12.4)$ & & \\
\hline \multicolumn{7}{|l|}{ Intrinsic subtypes } \\
\hline Luminal A & 149 & $64(43.0)$ & $64(43.0)$ & 21(14.1) & 0.683 & 0.595 \\
\hline Luminal B & 273 & $129(47.3)$ & $110(40.3)$ & $34(12.5)$ & & \\
\hline HER2-positive & 162 & $73(45.1)$ & $73(45.1)$ & $16(9.9)$ & & \\
\hline Triple-negative & 27 & $10(37.0)$ & $11(40.7)$ & $6(22.2)$ & & \\
\hline
\end{tabular}

Table 5. The association between SNP locus rs10958704 and clinicopathological characteristics of breast cancer. Note: ${ }^{a}$ Under dominate model, ${ }^{\mathrm{b}}$ under co-dominate model

\begin{tabular}{|c|c|c|c|c|c|c|}
\hline Clinico-pathological characteristics & Cases & $\mathrm{GG}(\%)$ & $\begin{array}{c}\text { rs10958704 } \\
\text { GA(\%) }\end{array}$ & $\mathrm{AA}(\%)$ & $\mathrm{Pa}$ & $\mathrm{Pb}$ \\
\hline \multicolumn{7}{|l|}{ TNM stage } \\
\hline I, II & 532 & $242(45.5)$ & $227(42.7)$ & $63(11.8)$ & 0.237 & 0.474 \\
\hline III & 143 & $73(51.0)$ & $56(39.2)$ & $14(39.2)$ & & \\
\hline \multicolumn{7}{|l|}{ Tumor size $(\mathrm{cm})$} \\
\hline$\leqslant 2$ & 583 & $268(46.0)$ & $250(42.9)$ & $65(11.1)$ & 0.365 & 0.513 \\
\hline$>2$ & 88 & $45(51.1)$ & $32(36.4)$ & $11(12.5)$ & & \\
\hline \multicolumn{7}{|l|}{ Pathological stage } \\
\hline I, II & 446 & $214(48.0)$ & $179(40.1)$ & $53(11.9)$ & 0.676 & 0.535 \\
\hline III & 120 & $55(45.8)$ & $54(45.0)$ & $11(9.2)$ & & \\
\hline \multicolumn{7}{|l|}{ LNM } \\
\hline Negative & 425 & $187(44.0)$ & $192(45.2)$ & $46(10.8)$ & 0.338 & 0.289 \\
\hline Positive & 294 & $140(47.6)$ & $116(39.5)$ & $38(12.9)$ & & \\
\hline \multicolumn{7}{|l|}{ ER status } \\
\hline Negative & 244 & $111(45.5)$ & $105(43.0)$ & $28(11.5)$ & 0.984 & 0.946 \\
\hline Positive & 447 & $203(45.4)$ & $189(42.3)$ & $55(12.3)$ & & \\
\hline \multicolumn{7}{|l|}{ PR status } \\
\hline Negative & 319 & $144(45.1)$ & $136(42.6)$ & $39(12.2)$ & 0.883 & 0.982 \\
\hline Positive & 372 & $170(45.7)$ & $158(42.5)$ & $44(11.8)$ & & \\
\hline \multicolumn{7}{|l|}{ HER2 status } \\
\hline Negative & 492 & $223(45.3)$ & $212(43.1)$ & $57(11.6)$ & 0.515 & 0.545 \\
\hline Positive & 119 & $50(42.0)$ & $51(42.9)$ & $18(15.1)$ & & \\
\hline \multicolumn{7}{|l|}{ Ki67 status } \\
\hline Negative & 258 & $103(39.9)$ & $118(45.7)$ & $37(14.3)$ & 0.023 & 0.056 \\
\hline Positive & 428 & $209(48.8)$ & $174(40.7)$ & $45(10.5)$ & & \\
\hline \multicolumn{7}{|l|}{ P53 status } \\
\hline Negative & 499 & $226(45.3)$ & $214(42.9)$ & $59(11.8)$ & 0.878 & 0.949 \\
\hline Positive & 185 & $85(45.9)$ & $77(41.6)$ & $23(12.4)$ & & \\
\hline \multicolumn{7}{|l|}{ Intrinsic subtypes } \\
\hline Luminal A & 149 & $63(42.3)$ & $65(43.6)$ & $21(14.1)$ & 0.676 & 0.755 \\
\hline Luminal B & 273 & $128(46.9)$ & $112(41.0)$ & $33(12.1)$ & & \\
\hline HER2-positive & 162 & $72(44.4)$ & $74(45.7)$ & $16(9.9)$ & & \\
\hline Triple-negative & 27 & $10(37.0)$ & $12(44.4)$ & $5(18.5)$ & & \\
\hline
\end{tabular}


receptor (PR), HER2, P53, Ki67, and intrinsic subtypes (Luminal A, Luminal B, HER2-positive, and Triplenegative) (Table 3-5).

According to the results, for SNP rs 17175624 and rs10958704, patients of mutant heterozygotes and homozygotes were more likely to have Ki67negative tumors under dominate model $(P=0.029$; $P=0.023$, respectively). However, no correlations were found for the two SNPs with other disease characteristics, including lymph node metastasis, tumor size, tumor grade, clinic stage, and the status of ER or PR, HER2, P53, and intrinsic subtypes. In addition, we did not find associations between rs17182023 and all disease characteristics.

\section{Association of FGFR1 genetic polymorphisms with FGFR1 protein expression}

A total of 348 patients were randomly selected from the 747 breast cancer cases for the detection of FGFR1 protein expression levels. The association between the three SNP loci and FGFR1 protein expression was shown in Table 6. For rs17182023,
Table 6. The correlations between FGFR1genetic polymorphisms and FGFR1 protein expression. Note: Data presented as the number (\% of total number)

\begin{tabular}{|c|c|c|c|c|c|c|}
\hline \multirow[b]{2}{*}{ SNP } & \multirow[b]{2}{*}{ Genotype } & \multirow[b]{2}{*}{$\mathrm{N}$} & \multicolumn{2}{|c|}{ FGFR1 protein expression } & \multirow[b]{2}{*}{$\chi^{2}$} & \multirow[b]{2}{*}{$\mathrm{P}$} \\
\hline & & & Low expression(\%) & High expression(\%) & & \\
\hline \multirow[t]{4}{*}{ rs17182023 } & GG & 153 & $47(34.8)$ & $106(49.8)$ & & \\
\hline & GA & 146 & $62(45.9)$ & $84(39.4)$ & & \\
\hline & AA & 49 & $26(19.3)$ & $23(10.8)$ & 9.231 & 0.010 \\
\hline & $\mathrm{GA}+\mathrm{AA}$ vs $\mathrm{GG}$ & 195 & $88(65.2)$ & $107(50.2)$ & 7.497 & 0.006 \\
\hline \multirow[t]{4}{*}{ rs17175624 } & CC & 169 & $73(54.1)$ & $96(45.1)$ & & \\
\hline & $\mathrm{CA}$ & 138 & $50(37.0)$ & $88(41.3)$ & & \\
\hline & AA & 41 & $12(8.9)$ & $29(13.6)$ & 3.327 & 0.189 \\
\hline & $\mathrm{CA}+\mathrm{AA}$ vs $\mathrm{CC}$ & 179 & $62(45.9)$ & $117(54.9)$ & 2.682 & 0.102 \\
\hline \multirow[t]{4}{*}{ rs10958704 } & GG & 169 & $72(53.3)$ & $97(45.5)$ & & \\
\hline & GA & 138 & $51(37.8)$ & $87(40.8)$ & & \\
\hline & AA & 41 & $12(8.9)$ & $29(13.6)$ & 2.796 & 0.247 \\
\hline & $\mathrm{GA}+\mathrm{AA}$ vs $\mathrm{GG}$ & 179 & $63(46.7)$ & $116(54.5)$ & 2.009 & 0.156 \\
\hline
\end{tabular}

Table 7. The correlations between FGFR1 protein expression and clinico-pathological characteristics of breast cancer

\begin{tabular}{|c|c|c|c|c|}
\hline \multirow{2}{*}{ Clinico-pathological characteristics } & \multirow{2}{*}{ Cases } & \multicolumn{2}{|c|}{$\begin{array}{l}\text { FGFR1 protein expression } \\
\end{array}$} & \multirow{2}{*}{$\mathrm{P}$} \\
\hline & & Low expression(\%) & High expression(\%) & \\
\hline \multicolumn{5}{|l|}{ TNM stage } \\
\hline I, II & 259 & $106(79.1)$ & 153(72.9) & 0.190 \\
\hline III & 85 & $28(20.9)$ & $57(27.1)$ & \\
\hline \multicolumn{5}{|l|}{ Tumor size $(\mathrm{cm})$} \\
\hline$\leqslant 2$ & 305 & $120(90.2)$ & $185(88.1)$ & 0.540 \\
\hline$>2$ & 38 & $13(9.8)$ & $25(11.9)$ & \\
\hline \multicolumn{5}{|l|}{ Pathological stage } \\
\hline I, II & 187 & $67(60.9)$ & $120(67.4)$ & 0.261 \\
\hline III & 101 & $43(39.1)$ & $58(32.6)$ & \\
\hline \multicolumn{5}{|l|}{ LNM } \\
\hline Negative & 182 & $80(61.5)$ & $102(49.3)$ & 0.028 \\
\hline Positive & 155 & $50(38.5)$ & $105(50.7)$ & \\
\hline \multicolumn{5}{|l|}{ ER status } \\
\hline Negative & 126 & $58(48.7)$ & $68(35.6)$ & 0.022 \\
\hline Positive & 184 & $61(51.3)$ & $123(64.4)$ & \\
\hline \multicolumn{5}{|l|}{ 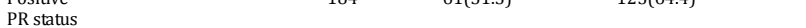 } \\
\hline Negative & 154 & $64(53.8)$ & $90(47.1)$ & 0.254 \\
\hline Positive & 156 & $55(46.2)$ & $101(52.9)$ & \\
\hline \multicolumn{5}{|l|}{ HER2 status } \\
\hline Negative & 252 & $104(94.5)$ & $148(86.0)$ & 0.024 \\
\hline Positive & 30 & $6(5.5)$ & $24(14.0)$ & \\
\hline \multicolumn{5}{|l|}{ Ki67 status } \\
\hline Negative & 96 & $36(30.5)$ & $60(31.7)$ & 0.820 \\
\hline Positive & 211 & $82(69.5)$ & $129(68.3)$ & \\
\hline \multicolumn{5}{|l|}{ P53 status } \\
\hline Negative & 214 & $79(66.9)$ & $135(71.8)$ & 0.367 \\
\hline \multirow{2}{*}{\multicolumn{5}{|c|}{$\begin{array}{l}\text { Posinve } \\
\text { Intrinsic Subtypes }\end{array}$}} \\
\hline & & & & \\
\hline Luminal A & 52 & $17(32.7)$ & $35(67.3)$ & 0.047 \\
\hline $\begin{array}{l}\text { Luminal B } \\
\text { Lumal }\end{array}$ & 137 & $47(34.3)$ & $90(65.7)$ & \\
\hline HER2-positive & 79 & $37(46.8)$ & $42(53.2)$ & \\
\hline Triple-negative & 14 & $9(64.3)$ & $5(35.7)$ & \\
\hline
\end{tabular}
the GA and AA genotypes conferred a significantly lower FGFR1 expression compared to the GG genotype under both dominant and co-dominant model $(P=0.010 ; P=0.006$, respectively). Furthermore, we observed a dose-dependent effect of the A allele; each additional copy increased the proportion of FGFR1 low expression patients. The negative correlation between the A allele and FGFR1 protein expression was confirmed by a Spearman rank correlation analysis $(r=-0.162, P=0.002)$. Nevertheless, no significant correlations were found between the other two SNP loci and the expression of FGFR1 protein.

\section{Association of FGFR1 expression with disease characteristics and prognosis of breast cancer}

In this part, we analyzed the effect of FGFR1 expression on disease characteristics and prognosis of breast cancer. Firstly, the association between clinico-pathological features and FGFR1 level was exhibited in Table 7. According to the results, patients with higher FGFR1 expression were more likely to have ER and HER2 positive breast cancer $(P=0.022 ; P=0.024$, respectively), and were prone to lymph node metastasis $(P=0.028)$. Besides, the results of intrinsic subtypes were also be of statistical significance, that patients with higher level of FGFR1 tended to have luminal type breast cancer $(P=0.047)$. There were no significant differences between FGFR1 protein expression and other disease characteristics. 
Next, the Cox proportional hazards model was employed to analyze the impact of specific factors on breast cancer prognosis, including lymph node metastasis, tumor size, tumorgrade, clinic stage, and the status of ER or PR, HER2, P53, Ki67, and FGFR1 protein expression (Table 8). The results showed that TNM stage, lymph node metastasis and FGFR1 expression were strongly correlated with the outcomes of breast cancer under univariate analysis, with risk ratios of $1.812(95 \% \mathrm{CI}=1.210$ 2.713, $P=0.004), 1.693(95 \%$ $\mathrm{CI}=1.219-2.351, P=0.002)$ and $1.587(95 \% \mathrm{CI}=1.055-2.386, P=$ 0.027), respectively. After that, a multivariate analysis was performed, the three prognostic factors which showed statistical significance above were involved. It was found that lymph node metastasis and FGFR1 expression were independent prognostic factors of breast cancer $(\mathrm{OR}=1.834,95 \% \mathrm{CI}=1.243-2.705, P=0.002$; $\mathrm{OR}=1.555,95 \% \mathrm{CI}=1.027-2.356, P=0.037$, respectively), while TNM stage did not show direct correlations to breast cancer prognosis. Besides, the overall survival curve are shown in Fig. 2. Patients with high FGFR1 protein expression were more likely to have poor outcomes $(P=$ $0.025)$.

\section{Discussion}

In this study, we focused on three SNP loci in FGFR1 gene, rs17182023, rs17175624 and rs10958704, and investigated their correlation with susceptibility of breast cancer, and their impact on FGFR1 protein expression. It was found that SNP rs17182023 was significantly associated with reduced risk of breast cancer, and had a certain connection with lower FGFR1 expression. Besides, we also confirmed that high FGFR1 expression was significantly correlated with poor prognosis.

FGFR1 is located at chromosome 8p12, and multiple studies have demonstrated that amplifications at this chromosomal region are found in up to $10 \%$ breast carcinomas, and are correlated to FGFR1 overexpression [18-21]. Likewise, the Cancer Genome Atlas (TCGA) showed that copy number gain / amplification of FGFR1 was around $10 \%$ in breast cancer [17]. The aberrant high expression of FGFR1 results in excessive activation of MAPK and PI3K-AKT signalling pathways [22, 23], which are strongly associated with oncogenesis and tumor progression. Recent studies have confirmed the correlations between FGFR1 amplification and breast cancer, and have provided important insights into the relationship between FGFR1 expression, histological subtype and survival. Turner et al. suggested that the amplification and overexpression of FGFR1 contributed to poor prognosis in luminaltype breast cancers, and drive anchorage-independent proliferation and endocrine therapy resistance [10]. Another research carried out by Elsheikh et al. also concluded that FGFR1 amplification was an independent predictor of breast cancer prognosis [9]. Our study showed that high FGFR1 protein expression was significantly associated with poor outcome 
in breast cancer patients, which was consistent with the previous studies. Based on these findings, further studies are warranted to determine the utility of FGFR1 expression analysis as a prognostic marker for breast cancer treatment.

FGFR1 amplification is frequent in luminal-type breast cancers $[9,10,24,25]$, and a number of studies have demonstrated that ER+ / FGFR1-amplified breast cancer cells and tumors retain proliferation and $\mathrm{ER} \alpha$ genomic activity despite estrogen deprivation therapy. It is reported that one third of FGFR1-amplified tumors also harbor amplification of CCND1, FGF3, FGF4, and FGF19 [26]. The FGF3/4/19 genes are located on chromosome 11q13, a region which also harbors $C C N D 1$, and is amplified in up to $15 \%$ of breast cancers [17]. This co-amplification has also been associated with resistance to estrogen deprivation in ER+ breast cancer and poor patient outcome. Interestingly, Hanker et al. found a copy number gains of FGF3/4/19 gene and evidence of increased FGFR signaling in HER2-amplified cell lines [27]. Preclinical and clinical evidence indicated that ER+ tumors with HER2 amplification responded poorly to endocrine therapy, particularly tamoxifen, probably due to the crosstalk between ER and HER2 signaling pathways $[28,29]$. With the activation of HER2 signalling, tamoxifen may lose its estrogen antagonist activity and may acquire more agonist-like activity, resulting in tumor growth stimulation [30]. In this study, we observed that patients with higher FGFR1 protein expression were more likely to have ER+ / HER2+ breast cancers, and this result showed the links between FGFR1 overexpression and HER2 amplification. Combining with the above, this might explain why FGFR1 overexpression contributes to endocrine therapy resistance to some extent.

SNP rs17182023 is located in the intronic region, near the 5' end of FGFR1 gene. To date, the biological function of this locus remains unclear, and there's few reports about its influence on breast cancer. Our study revealed that SNP rs17182023 in FGFR1 gene was strongly associated with breast cancer susceptibility, and interestingly, it showed a tendency of reducing the risk. Furthermore, we also found a correlation between the SNP locus and FGFR1 protein expression, that the patients with mutant genotypes had a lower FGFR1 level. It had been reported that some intronic variants could effect the expression of coding genes, via exon deletion, activation of the recessive splice sites and (or) the change of shear effect [31]. Since rs17182023 was located in intron region of FGFR1, it might effect FGFR1 protein expression in these ways. Therefore, we proposed a possibility that SNP rs17182023 could act as a protective factor on reducing breast cancer risk, via declining FGFR1 protein expression. This might provide a new sight to understand the association of genetic polymorphisms with breast tumorigenesis, but further studies are still required to illuminate the mechanisms. Additionally, we did not find associations of SNP rs17175624 and rs10958704 with the risk of breast cancer. Although patients with mutant genotypes of these two SNPs were more likely to have Ki67-negative tumors under dominate model according to the results, this could hardly be considered as a result of significance.

In conclusion, high FGFR1 protein expression was an independent risk factor of breast cancer, and implicated to poor prognosis. We evaluated three SNP loci, rs17182023, rs17175624 and rs10958704 in FGFR1 gene, and revealed the association between rs17182023 and the reduction of breast cancer risk. Moreover, SNP rs17182023 also correlated to FGFR1 protein expression, and its negative effect on FGFR1 expression may be a protective factor on breast cancer. Although this study might provide new insights to understand the relationships between breast carcinogenesis and FGFR1, and might contributes to the prognostic evaluation for breast cancer patients, these results still await further confirmation by ethnicity-matched larger studies.

\section{Acknowledgements}

We are grateful to all participants of this study. This study was supported by the National Natural Science Foundation of China (Yongdong Jiang, Grant No. 81202075), Heilongjiang Postdoctoral Fund, China (Yongdong Jiang, Grant No. LBH-Z13158), and the China Postdoctoral Science Fund (Yongdong Jiang, Grant No. 2015M571445). 


\section{Cellular Physiology Cell Physiol Biochem 2018;47:2569-2578

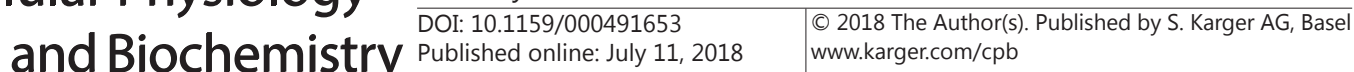

Wu et al.: FGFR1 Polymorphisms Affect Breast Cancer Risk

\section{Disclosure Statement}

The authors declare that they have no conflict of interests. The manuscript has been read and approved by all named authors.

\section{References}

1 Siegel RL, Miller KD, Jemal A: Cancer statistics, 2016 CA Cancer J Clin 2016;66:7-30.

2 Kimelman D, Kirschner M: Synergistic induction of mesoderm by FGF and TGF-beta and the identification of an mRNA coding for FGF in the early Xenopus embryo. Cell 1987;51:869-877.

$>3$ De Moerlooze L, Spencer-Dene B, Revest JM, Hajihosseini M, Rosewell I, Dickson C: An important role for the IIIb isoform of fibroblast growth factor receptor 2 (FGFR2) in mesenchymal-epithelial signalling during mouse organogenesis. Development 2000;127:483-492.

4 Beenken A, Mohammadi M: The FGF family: biology, pathophysiology and therapy. Nat Rev Drug Discov 2009;8:235-253.

5 Turner N, Grose R: Fibroblast growth factor signalling: from development to cancer. Nat Rev Cancer 2010;10:116-129.

6 Dienstmann R, Rodon J, Prat A, Perez-Garcia J, Adamo B, Felip E, Cortes J, Iafrate AJ, Nuciforo P, Tabernero J: Genomic aberrations in the FGFR pathway: opportunities for targeted therapies in solid tumors. Ann Oncol 2014;25:552-563.

7 Easton DF, Pooley KA, Dunning AM, Pharoah PD, Thompson D, Ballinger DG, Struewing JP, Morrison J, Field H, Luben R, Wareham N, Ahmed S, Healey CS, Bowman R; SEARCH collaborators, Meyer KB, Haiman CA, Kolonel LK, Henderson BE, Le Marchand L, et al.: Genome-wide association study identifies novel breast cancer susceptibility loci. Nature 2007;447:1087-1093.

-8 Hunter DJ, Kraft P, Jacobs KB, Cox DG, Yeager M, Hankinson SE, Wacholder S, Wang Z, Welch R, Hutchinson A, Wang J, Yu K, Chatterjee N, Orr N, Willett WC, Colditz GA, Ziegler RG, Berg CD, Buys SS, McCarty CA, Feigelson HS, Calle EE, Thun MJ, Hayes RB, Tucker M, Gerhard DS, Fraumeni JF Jr, Hoover RN, Thomas G, Chanock SJ: A genome-wide association study identifies alleles in FGFR2 associated with risk of sporadic postmenopausal breast cancer. Nat Genet 2007;39:870-874.

-9 Elbauomy Elsheikh S, Green AR, Lambros MB, Turner NC, Grainge MJ, Powe D, Ellis IO, Reis-Filho JS: FGFR1 amplification in breast carcinomas: a chromogenic in situ hybridisation analysis. Breast Cancer Res 2007;9: R23.

10 Turner N, Pearson A, Sharpe R, Lambros M, Geyer F, Lopez-Garcia MA, Natrajan R, Marchio C, Iorns E, Mackay A, Gillett C, Grigoriadis A, Tutt A, Reis-Filho JS, Ashworth A: FGFR1 amplification drives endocrine therapy resistance and is a therapeutic target in breast cancer. Cancer Res 2010;70:2085-2094.

11 Xian W, Pappas L, Pandya D, Selfors LM, Derksen PW, de Bruin M, Gray NS, Jonkers J, Rosen JM, Brugge JS: Fibroblast growth factor receptor 1-transformed mammary epithelial cells are dependent on RSK activity for growth and survival. Cancer Res 2009;69:2244-2251.

12 Xian W, Schwertfeger KL, Vargo-Gogola T, Rosen JM: Pleiotropic effects of FGFR1 on cell proliferation, survival, and migration in a 3D mammary epithelial cell model. Cell Biol 2005;171:663-673.

13 Agarwal D, Pineda S, Michailidou K, Herranz J, Pita G, Moreno LT, Alonso MR, Dennis J, Wang Q, Bolla MK, Meyer KB, Menéndez-Rodríguez P, Hardisson D, Mendiola M, González-Neira A, Lindblom A, Margolin S, Swerdlow A, Ashworth A, Orr N, Jones M, et al.: FGF receptor genes and breast cancer susceptibility: results from the Breast Cancer Association Consortium. Br J Cancer 2014;110:1088-1100.

14 Koole K, Brunen D, van Kempen PM, Noorlag R, de Bree R, Lieftink C, van Es RJ, Bernards R, Willems SM: FGFR1 Is a Potential Prognostic Biomarker and Therapeutic Target in Head and Neck Squamous Cell Carcinoma. Clin Cancer Res 2016;22:3884-3893.

15 Hammond ME, Hayes DF, Dowsett M, Allred DC, Hagerty KL, Badve S, Fitzgibbons PL, Francis G, Goldstein NS, Hayes M, Hicks DG, Lester S, Love R, Mangu PB, McShane L, Miller K, Osborne CK, Paik S, Perlmutter J, Rhodes A, Sasano H, Schwartz JN, Sweep FC, Taube S, Torlakovic EE, Valenstein P, Viale G, Visscher D, Wheeler T, Williams RB, Wittliff JL, Wolff AC: American Society of Clinical Oncology/College Of American Pathologists guideline recommendations for immunohistochemical testing of estrogen and progesterone receptors in breast cancer. J Clin Oncol 2010;28:2784-2795. 


\section{Cellular Physiology Cell Physiol Biochem 2018;47:2569-2578 \begin{tabular}{l|l} 
and Biochemistry Publisned onlIne: July $1 \mathrm{I}, 2018$ & $\begin{array}{l}\text { () } 2018 \text { The Author(s). Published by S. Karger AG, Basel } \\
\text { www.karger.com/cpb }\end{array}$ \\
\hline
\end{tabular}}

Wu et al.: FGFR1 Polymorphisms Affect Breast Cancer Risk

-16 Hsu CY, Ho DM, Yang CF, Lai CR, Yu IT, Chiang H: Interobserver reproducibility of Her-2/neu protein overexpression in invasive breast carcinoma using the DAKO HercepTest. Am J Clin Pathol 2002;118:693698.

17 Cancer Genome Atlas Network: Comprehensive molecular portraits of human breast tumours. Nature 2010;490:61-70.

18 Gelsi-Boyer V, Orsetti B, Cervera N, Finetti P, Sircoulomb F, Rougé C, Lasorsa L, Letessier A, Ginestier C, Monville F, Esteyriès S, Adélaïde J, Esterni B, Henry C, Ethier SP, Bibeau F, Mozziconacci MJ, CharafeJauffret E, Jacquemier J, Bertucci F, Birnbaum D, Theillet C, Chaffanet M: Comprehensive profiling of 8p1112 amplification in breast cancer. Mol Cancer Res 2005;3:655-667.

-19 Andre F, Job B, Dessen P, Tordai A, Michiels S, Liedtke C, Richon C, Yan K, Wang B, Vassal G, Delaloge S, Hortobagyi GN, Symmans WF, Lazar V, Pusztai L: Molecular characterization of breast cancer with highresolution oligonucleotide comparative genomic hybridization array. Clin Cancer Res 2009;15:441-451.

20 Chin K, DeVries S, Fridlyand J, Spellman PT, Roydasgupta R, Kuo WL, Lapuk A, Neve RM, Qian Z, Ryder T, Chen F, Feiler H, Tokuyasu T, Kingsley C, Dairkee S, Meng Z, Chew K, Pinkel D, Jain A, Ljung BM, Esserman L, Albertson DG, Waldman FM, Gray JW: Genomic and transcriptional aberrations linked to breast cancer pathophysiologies. Cancer Cell 2006;10:529-541.

21 Reis-Filho JS, Simpson PT, Turner NC, Lambros MB, Jones C, Mackay A, Grigoriadis A, Sarrio D, Savage K, Dexter T, Iravani M, Fenwick K, Weber B, Hardisson D, Schmitt FC, Palacios J, Lakhani SR, Ashworth A: FGFR1 emerges as a potential therapeutic target for lobular breast carcinomas. Clin Cancer Res 2006;12:6652-6662.

22 Eswarakumar VP, Lax I, Schlessinger J: Cellular signaling by fibroblast growth factor receptors. Cytokine Growth Factor Rev 2005;16:139-149.

23 Altomare DA, Testa JR: Perturbations of the AKT signaling pathway in human cancer. Oncogene 2005;24:7455-7464.

-24 Stephens PJ, Tarpey PS, Davies H, Van Loo P, Greenman C, Wedge DC, Nik-Zainal S, Martin S, Varela I, Bignell GR, Yates LR, Papaemmanuil E, Beare D, Butler A, Cheverton A, Gamble J, Hinton J, Jia M, Jayakumar A, Jones D, Latimer C, Lau KW, McLaren S, McBride DJ, Menzies A, Mudie L, Raine K, Rad R, Chapman MS, Teague J, Easton D, Langerød A; Oslo Breast Cancer Consortium (OSBREAC), Lee MT, Shen CY, Tee BT, Huimin BW, Broeks A, Vargas AC, Turashvili G, Martens J, Fatima A, Miron P, Chin SF, Thomas G, Boyault S, Mariani O, Lakhani SR, van de Vijver M, van 't Veer L, Foekens J, Desmedt C, Sotiriou C, Tutt A, Caldas C, Reis-Filho JS, Aparicio SA, Salomon AV, Børresen-Dale AL, Richardson AL, Campbell PJ, Futreal PA, Stratton MR: The landscape of cancer genes and mutational processes in breast cancer. Nature 2012;486:400-404.

25 Tomiguchi M, Yamamoto Y, Yamamoto-Ibusuki M, Goto-Yamaguchi L, Fujiki Y, Fujiwara S, Sueta A, Hayashi M, Takeshita T, Inao T, Iwase H: Fibroblast growth factor receptor-1 protein expression is associated with prognosis in estrogen receptor-positive/human epidermal growth factor receptor-2-negative primary breast cancer. Cancer Sci 2016;107:491-498.

26 Cuny M, Kramar A, Courjal F, Johannsdottir V, Iacopetta B, Fontaine H, Grenier J, Culine S, Theillet C: Relating genotype and phenotype in breast cancer: an analysis of the prognostic significance of amplification at eight different genes or loci and of p53 mutations. Cancer Res 2000;60:1077-1083.

-27 Hanker AB, Garrett JT, Estrada MV, Moore PD, Ericsson PG, Koch JP, Langley E, Singh S, Kim PS, Frampton GM, Sanford E, Owens P, Becker J, Groseclose MR, Castellino S, Joensuu H, Huober J, Brase JC, Majjaj S, Brohée S, Venet D, Brown D, Baselga J, Piccart M, Sotiriou C Arteaga CL: HER2-Overexpressing Breast Cancers Amplify FGFR Signaling upon Acquisition of Resistance to Dual Therapeutic Blockade of HER2 Clin Cancer Res 2017;23:4323-4334.

28 Arpino G, Gutierrez C, Weiss H, Rimawi M, Massarweh S, Bharwani L, De Placido S, Osborne CK, Schiff R: Treatment of human epidermal growth factor receptor 2-overexpressing breast cancer xenografts with multiagent HER-targeted therapy. J Natl Cancer Inst 2007;99:694-705.

29 Arpino G, Wiechmann L, Osborne CK, Schiff R: Crosstalk between the estrogen receptor and the HER tyrosine kinase receptor family: molecular mechanism and clinical implications for endocrine therapy resistance. Endocr Rev 2008;29:217-233.

-30 Osborne CK, Shou J, Massarweh S, Schiff R: Crosstalk between estrogen receptor and growth factor receptor pathways as a cause for endocrine therapy resistance in breast cancer. Clin Cancer Res 2005;11:865s-870s.

-31 Copertino DW, Hallick RB: Group II and group III introns of twintrons: potential relationships with nuclear pre-mRNA introns. Trends Biochem Sci 1993;18:467-471. 